Cite this: Soft Matter, 2014, 10, 3009

Received 16th January 2014

Accepted 19th February 2014

DOI: $10.1039 / c 4 s m 00114 a$

www.rsc.org/softmatter

\section{The effects of pressure and temperature on the energetics and pivotal surface in a monoacylglycerol/water gyroid inverse bicontinuous cubic phase $\uparrow$}

\author{
T.-Y. Dora Tang, ${ }^{\text {*ab }}$ Annela M. Seddon, ${ }^{\text {ac }}$ Christoph Jeworrek, ${ }^{d}$ Roland Winter, ${ }^{d}$ \\ Oscar Ces, ${ }^{a}$ John M. Seddon ${ }^{a}$ and Richard H. Templer ${ }^{a}$
}

\begin{abstract}
We have studied the effect of pressure and temperature on the location of the pivotal surface in a lipid inverse bicontinuous gyroid cubic phase $\left(Q_{11}^{G}\right)$, described by the area at the pivotal surface $\left(A_{n}\right)$, the volume between the pivotal surface and the bilayer midplane $\left(V_{n}\right)$, and the molecular volume of the lipid (V). Small angle X-ray scattering (SAXS) was used to measure the swelling behaviour of the lipid, monolinolein, as a function of pressure and temperature, and the data were fitted to two different geometric models: the parallel interface model (PIM), and the constant mean curvature model (CMCM). The results show that an increase in temperature leads to a shift in the location of the pivotal surface towards the bilayer midplane, whilst an increase in pressure causes the pivotal surface to move towards the interfacial region. In addition, we describe the relevance of $A_{n}, V_{n}$ and $V$ for modeling the energetics of curved mesophases with specific reference to the mean curvature at the pivotal surface and discuss the significance of this parameter for modelling the energetics of curved mesophases.
\end{abstract}

\section{Introduction}

Liquid crystalline phases with curved interfaces, such as the inverse bicontinuous cubic phases $\left(\mathrm{Q}_{\mathrm{II}}\right)$, are mathematically and geometrically elegant. The most well known and characterized of the inverse bicontinuous phases are based upon the diamond, gyroid and primitive triply periodic minimal surfaces., ${ }^{\mathbf{1 , 2}}$ In the liquid crystalline phase $\mathrm{Q}_{\mathrm{II}}^{\mathrm{D}}$ (diamond), $\mathrm{Q}_{\mathrm{II}}^{\mathrm{G}}$ (gyroid) and $\mathrm{Q}_{\mathrm{II}}^{\mathrm{P}}$ (primitive) the midplane of an amphiphilic bilayer lies upon a diamond, gyroid or primitive minimal surface of zero mean curvature and subdivides three dimensional space to form intertwined but non-penetrating water channels. Each inverse bicontinuous cubic phase differs in the connectivity of these water channels, for example, in the $\mathrm{Q}_{\mathrm{II}}^{\mathrm{G}}$ phase the channels are connected 3-fold at $120^{\circ}$ (Fig. 1), whilst the junctions in the $\mathrm{Q}_{\mathrm{II}}^{\mathrm{D}}$ phase connect 4 -fold at $109^{\circ}$ and 6-fold at $90^{\circ}$ for the $\mathrm{Q}_{\mathrm{II}}^{\mathrm{P}}$ phase.

The formation of these curved mesophases from amphiphilic molecules such as surfactants, polymers and lipids are

${ }^{a}$ Department of Chemistry, Imperial College London, Exhibition Road, London, SW7 $2 A Y, U K$

${ }^{b}$ School of Chemistry, University of Bristol, Cantock's Close, Bristol BS8 1TS, UK. E-mail: chtydt@bristol.ac.uk

${ }^{c} H H$ Wills Physics Laboratory, University of Bristol, Tyndall Avenue, BS8 1FD, UK ${ }^{d}$ Physical Chemistry I-Biophysical Chemistry, Faculty of Chemistry, Technische Universität Dortmund, Otto-Hahn-Str. 6, 44227 Dortmund, Germany

$\dagger$ Electronic supplementary information (ESI) available: See DOI: $10.1039 / \mathrm{c} 4 \mathrm{sm} 00114 \mathrm{a}$ driven by (i) the hydrophobic effect, ${ }^{3}$ and (ii) a balance between the curvature elastic stress and packing energy. ${ }^{4}$ When curvature elastic stress drives the deformation of a flat bilayer to induce curvature there is a large energetic cost associated with the formation of hydrophobic voids which can be compensated by the stretching of hydrocarbon chains within the bilayer. The balance of curvature stress and packing energy leads to an energy minimum which favours the stable formation of the inverse bicontinuous phases. Experimentally it has been shown that the hydration, temperature, pressure, $\mathrm{pH}$ and the molecular structure of the lipid ${ }^{5-12}$ can tune the formation of the $\mathrm{Q}_{\mathrm{II}}$ phases. As a consequence, these structures have been exploited in technologies ranging from drug delivery ${ }^{\mathbf{1 1 , 1 3 , 1 4}}$ and renewable energies $^{7,15-17}$ to protein crystallography ${ }^{\mathbf{1 2 , 1 8 - 2 0}}$ and gene silencing $^{21}$ where the large surface area to volume ratio, high water content and controllable and tuneable structural properties have been exploited. However, a complete theoretical and energetic description of inverse bicontinuous phases is still lacking and this is essential for rational design of curved mesophases for technological applications.

One of the difficulties in modeling the $\mathrm{Q}_{\mathrm{II}}$ phases is attributed to its curved bilayer as the curvature and therefore the energetics of the phase are dependent on the transverse location within the bilayer from which it is measured..$^{22}$ However, within the curved bilayer there exists a surface, the pivotal surface, whose area remains constant as the bilayer bends with the swelling of the unit cell, thus the energy can be defined at 
(a)

(b)

(c)

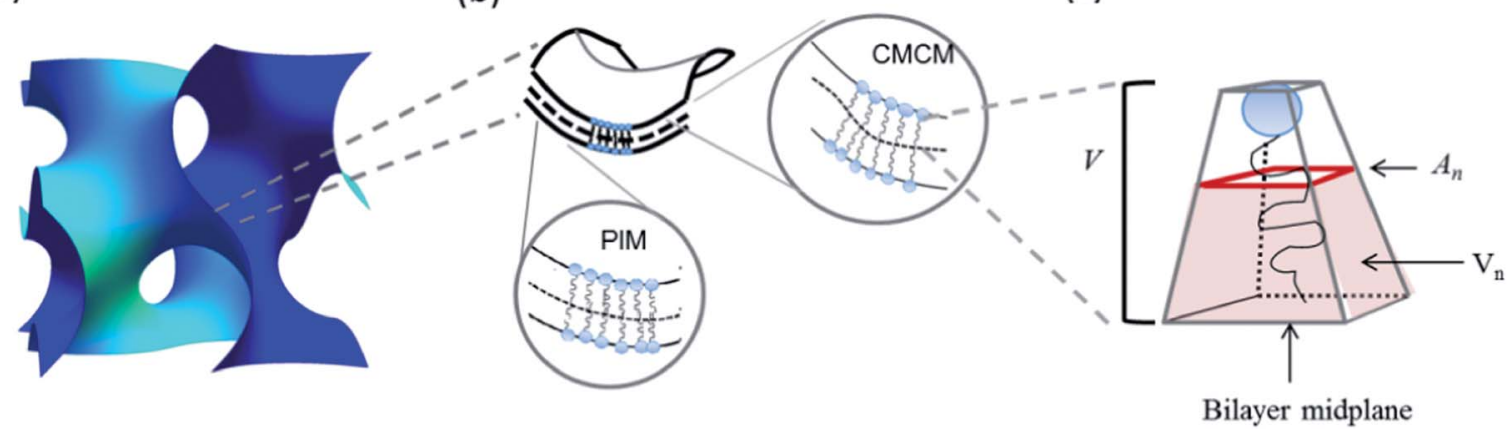

Fig. 1 (a) Gyroid minimal surface which the $Q_{\| l}^{G}$ phase is based on. (b) Cartoon to show the geometric differences in the parallel interface model (PIM) and the constant mean curvature model (CMCM). In the PIM the bilayer is constrained to be equidistant and normal to the bilayer midplane and in the CMCM the bilayer is constrained to adopt a constant mean curvature interface. (c) Cartoon depicting the pivotal surface parameters $V_{n}$, $A_{n}$ and $V$ for a lipid.

this surface and its location can be obtained by modeling isothermal and isobaric swelling data. ${ }^{23,24}$

The pivotal surface of a monolayer is defined by three parameters; $A_{\mathrm{n}}$, the area at the pivotal surface; $V_{\mathrm{n}}$, the volume between the bilayer midplane and the pivotal surface; and $V$, the molecular volume (Fig. 1c). $\left(\frac{V_{\mathrm{n}}}{V}\right)$ is directly related to the position of the pivotal surface in the lipid bilayer. $V$ can be experimentally obtained by measuring the density of the lipid whilst $A_{\mathrm{n}}$ and $V_{\mathrm{n}}$ are found by fixing the geometry of the interface. For the case of the inverse bicontinuous cubic phases, this interface can be defined by two opposing geometric models (Fig. 1b). The first (historically) is the parallel interface model (PIM) ${ }^{25}$ where the pivotal surface is constrained to lie equidistant from the bilayer midplane. In the second geometry, the Constant Mean Curvature Model (CMCM), the interface adopts a constant mean curvature. ${ }^{26-28}$ Detailed theory behind each of these models is given in Appendix A. Constraining the interface inevitably leads to molecular frustration within the bilayer; for example, curvature stress will dominate when a monolayer is constrained to a parallel interface. Conversely, packing frustration will dominate when the bilayers are constrained to a constant mean curvature. Therefore, a balance of energetic contributions from packing frustration and curvature stress is believed to lead to the stable formation of the inverse bicontinuous cubic phases; ${ }^{29}$ however the exact contributions from frustration and stress are not fully understood and this is a non-trivial matter.

Previous studies have shown that the monoacylglycerols form a range of inverse bicontinuous cubic phases as a function of pressure and temperature ${ }^{6,7,11,30,31}$ and their well characterized experimental phase behavior make them ideal models for measuring the pivotal surface parameters. However, the PIM and the CMCM are limited at low water contents where the molecular deformations within the monolayer increase and the bilayer can no longer maintain a fixed geometry. As the $\mathrm{Q}_{\mathrm{II}}^{\mathrm{G}}$ phase formed by monolinolein is hydrated between $15 \mathrm{wt} \%$ water and $30 \mathrm{wt} \%$ water, determining the pivotal surface parameters in this system should not be affected by the limits of the geometric models.
Here we use the two geometric models, the PIM and the CMCM, to model the location of the pivotal surface in the $\mathrm{Q}_{\mathrm{II}}^{\mathrm{G}}$ phase, as a function of pressure and temperature, in the binary monolinolein (ML)-water system. The swelling behavior of ML as a function of temperature and pressure was characterized using SAXS, and $A_{\mathrm{n}}$ and $V_{\mathrm{n}}$ were obtained from fitting PIM and CMCM to the swelling data. Having obtained $A_{\mathrm{n}}$ and $V_{\mathrm{n}}$ the mean curvature at the pivotal surface was calculated using the constant mean curvature model, and the validity of the geometrical models for obtaining structural parameters in curved lipid bilayers is discussed.

\section{Materials and methods}

\section{Sample preparation}

Lyophilised monolinolein (ML) (Larodan, Switzerland) was mixed with a known amount of HPLC grade water (Sigma Aldrich, UK) to the desired wt $\%$ water. The weight of the lipid and water was measured to a precision of 6 decimal places. Sample homogeneity was achieved by subjecting the lipid-water mixture to at least 50 freeze-thaw cycles $\left(-70{ }^{\circ} \mathrm{C}\right.$ to $\left.20^{\circ} \mathrm{C}\right)$.

For temperature dependent experiments, samples were prepared directly into $1.5 \mathrm{~mm}$ special glass capillaries. Once the lipid and water were loaded into the glass capillary the capillary was heat sealed and further sealed with silicone sealant and subjected to freeze-thaw cycles. These were stored at $-20{ }^{\circ} \mathrm{C}$ until the experiments were carried out. For pressure dependent experiments the samples were prepared in glass vials as described above and stored at $-20{ }^{\circ} \mathrm{C}$. Immediately before the experiment the lipid-water mixture was transferred to a Teflon ring enclosed by two mylar foils which isolated the sample from the external environment. The deviation from the weighed water content and the actual water content was typically $1 \%$; this error is attributed to water loss during sample transfer to the Teflon spacers and is typical of this experimental procedure.

\section{X-ray scattering measurements}

Temperature dependent experiments were undertaken using an in-house Bede Microscource ${ }^{\mathrm{TM}} \mathrm{X}$-ray generator (Durham, 
UK). Temperature was controlled by an external water bath; the temperature of the sample holder is controlled via a Pt100 (TC limited, Uxbridge, UK) temperature probe connected to a microcontroller which has the capability of maintaining the temperature to $\pm 0.1{ }^{\circ} \mathrm{C}$. X-ray diffraction images were acquired using an image intensified chargecoupled device X-ray camera (Gemstar HS, Photonic Science Ltd, UK). Typically, five 30 second exposures were taken at each temperature and summed using the custom built software package AXcess. ${ }^{32}$ Images were acquired at $2{ }^{\circ} \mathrm{C}$ intervals from $0{ }^{\circ} \mathrm{C}$ to $70{ }^{\circ} \mathrm{C}$ with a 10 minute equilibration at each temperature. Lattice parameter values (in $\AA$ ) were calibrated using silver behenate ( $a=58.38 \AA$ ) and AXcess was used to characterise the phase and to determine the lattice parameter to an accuracy of $\pm 0.05 \AA$.

Pressure dependent experiments were performed at beamline ID02 at the European Synchroton Radiation Facility (ESRF), Grenoble, France and I22 at Diamond Light Source (DLS), Oxfordshire, UK using a high pressure diamond cell ${ }^{16}$ constructed from Ni-Cr-Co alloy (NIMONIC 90) capable of withstanding hydrostatic pressures of up to 3000 bar generated by a manual pumping system. The pump and high pressure tubing and valves were purchased from Nova Swiss, Effretikon, Switzerland. Temperature was controlled via circulating water from an external water bath (accurate \pm 0.2 $\left.{ }^{\circ} \mathrm{C}\right)$ through the jacket of the cell and measured by a thermocouple embedded into the body of the cell. The high pressure cell is equipped with diamond windows glued into metal supports and X-ray transmission through the diamond windows was approximately 65\% using X-rays of energy 17 $\operatorname{keV}(\lambda=0.75 \AA)$. At beamline ID02, ESRF, a maximum flux of $4 \times 10^{13}$ photons per second was achieved. To achieve sample equilibration the samples were subjected to 10 pressure cycles from 1 bar to 2000 bar and samples were left for approximately 30 minutes after a change in temperature, and at least $2 \mathrm{~min}$ at each pressure before capturing a X-ray diffraction image with typical exposure times of $0.1 \mathrm{~s}$. X-ray diffraction images were taken using a Frelon (Fast readout, low noise) Kodak CCD detector with a maximum frame rate of 15 frames per s. Diffraction patterns were analyzed off line using AXcess software.

\section{Lipid density measurements}

The density of limited hydration monolinolein, over a range of temperatures and pressures, was measured using an Anton Paar DMA 60/512P (Graz Austria) vibrating tube densimeter with a steel U-tube of internal volume $2.5 \mathrm{ml}$ connected to a high pressure network capable of achieving pressures up to $700 \mathrm{bar}$. Temperature was controlled via a recirculating water bath, accurate to $0.1^{\circ} \mathrm{C}$.

Three $1 \mathrm{~g}$ monolinolein-water samples were prepared with 23 wt\% water using degassed Milli-Q water. Samples were mechanically mixed via centrifugation and then subjected to more than 40 freeze-thaw cycles to ensure sample homogeneity. The individual portions were combined and freeze-thawed a further 20 times, then stored under nitrogen at $-20{ }^{\circ} \mathrm{C}$ until required. Samples were loaded into the U-tube using a custom built apparatus to minimize the number of air bubbles in the sample. Lipid density was measured at $11^{\circ} \mathrm{C}, 17^{\circ} \mathrm{C}, 24^{\circ} \mathrm{C}, 37^{\circ} \mathrm{C}$ from 0-700 bar at 100 bar intervals.

To obtain an accurate water content of the lipid-water mixture the sample was removed from the U-tube after the experiment, weighed, and then re-weighed after 48 hours of lyophilisation. There was a $1 \%$ difference in water content compared to the expected water content, which was attributed to lipid loss during mechanical mixing or sample transfer.

The molecular volume was obtained from the lipid density as a function of pressure and temperature. Molecular volumes at intermediate temperatures and pressures were obtained via extrapolation of the experimental data (Table 1 ESI $\dagger$ ).

\section{Modelling the energetics of the inverse bicontinuous phases using geometric models}

Determining the properties of the pivotal surface using the parallel interface model. This model is embodied in eqn (1) ${ }^{33}$ where $\left\langle A_{\mathrm{n}}\right\rangle$ and $\left\langle V_{\mathrm{n}}\right\rangle$ are the surface averaged molecular area at the pivotal surface and the average molecular volume between the pivotal surface and the bilayer midplane respectively. $V$ is the experimentally determined molecular volume, $a$ is the lattice parameter of the mesophase, $\phi_{\mathrm{w}}$ is the water volume fraction of the bulk mesophase defined by eqn (2), $\sigma$ is the dimensionless surface area of the inverse bicontinuous phase, and $\chi$ is the Euler characteristic. For $\mathrm{Q}_{\mathrm{II}}^{\mathrm{G}}, \sigma=3.0915$ and $\chi=-8$.

$$
\begin{aligned}
& a=\frac{\left\langle V_{\mathrm{n}}\right\rangle}{\left\langle A_{\mathrm{n}}\right\rangle\left(1-\phi_{\mathrm{w}}\right)}\left(-2 \sigma+\frac{2^{\frac{5}{3}} \sigma^{2}}{\left(4 \sigma^{3}+9 \pi \chi\left(1-\phi_{\mathrm{w}}\right)^{2}\left(\frac{\left\langle V_{\mathrm{n}}\right\rangle}{\langle V\rangle}\right)^{2}+3\left(1-\phi_{\mathrm{w}}\right)\left(\frac{\left\langle V_{\mathrm{n}}\right\rangle}{\langle V\rangle}\right)^{\left.\pi \chi\left(8 \sigma^{3}+9 \pi \chi\left(1-\phi_{\mathrm{w}}\right)^{2}\left(\frac{\left\langle V_{\mathrm{n}}\right\rangle}{\langle V\rangle}\right)^{2}\right)\right)^{\frac{1}{3}}}+2^{\frac{1}{3}}\right.}\right. \\
& \left./\left(4 \sigma^{3}+9 \pi \chi\left(1-\phi_{\mathrm{w}}\right)^{2}\left(\frac{\left\langle V_{\mathrm{n}}\right\rangle}{\langle V\rangle}\right)^{2}+3\left(1-\phi_{\mathrm{w}}\right)\left(\frac{\left\langle V_{\mathrm{n}}\right\rangle}{\langle V\rangle}\right) \sqrt{\pi \chi\left(8 \sigma^{3}+9 \pi \chi\left(1-\phi_{\mathrm{w}}\right)^{2}\left(\frac{\left\langle V_{\mathrm{n}}\right\rangle}{\langle V\rangle}\right)^{2}\right)}\right)^{\frac{1}{3}}\right)
\end{aligned}
$$




$$
\phi_{\mathrm{w}}=\frac{C_{\mathrm{w}}}{C_{\mathrm{w}}+\left(\left(1-C_{\mathrm{w}}\right)\left(\frac{\rho_{\mathrm{w}}}{\rho_{\mathrm{l}}}\right)\right)}
$$

Determining the properties of the pivotal surface using the Constant Mean Curvature Model (CMCM). The constant mean curvature model is embodied in eqn (3), where the total volume fraction occupying the region between the pivotal surface and the bilayer midplane is given by $\phi_{\mathrm{n}}=\frac{V_{\mathrm{n}}}{V}\left(1-\phi_{\mathrm{w}}\right) \cdot{ }^{33}$ Relating the volume fraction $\phi_{\mathrm{w}}, V, A_{\mathrm{n}}$ and $V_{\mathrm{n}}$ to the lattice parameter, $a$, the constant mean curvature model is given for $\mathrm{Q}_{\mathrm{II}}^{\mathrm{G}}$ by eqn (3), with $\sigma_{0}=3.0915 ; \sigma_{1}=-1.3317 ; \sigma_{2}=-0.19974 ; \sigma_{3}=-0.80113$.

$$
a=2 \sum_{i=0} \frac{\sigma_{i}\left[\left(\frac{V_{\mathrm{n}}}{V}\right)\left(1-\phi_{\mathrm{w}}\right)\right]^{2 i}}{\left(\frac{A_{\mathrm{n}}}{V}\right)\left(1-\phi_{\mathrm{w}}\right)}
$$

The curvature elastic energy determined using the constant mean curvature model. The curvature elastic energy, $\mu_{\mathrm{c}}$, for a monolayer is given by the Helfrich Hamiltonian:

$$
\mu_{\mathrm{c}}=2 \kappa A_{\mathrm{n}}\left(H_{\mathrm{n}}-H_{0}\right)^{2}+\kappa_{\mathrm{G}} A_{\mathrm{n}} K_{\mathrm{n}}
$$

where $\kappa$ is the mean curvature modulus, $A_{\mathrm{n}}$ is the cross-sectional area of the lipid at the pivotal surface, $H_{\mathrm{n}}$ and $K_{\mathrm{n}}$ are the mean and Gaussian curvatures, $H_{0}$ is the spontaneous mean curvature, and $\kappa_{\mathrm{G}}$ is the Gaussian curvature modulus. The curvature elastic energy is dependent on the difference between the mean curvature at the pivotal surface and the spontaneous mean curvature and consequently the pivotal surface, which is defined by $A_{\mathrm{n}}, V_{\mathrm{n}}$ and $V$.

$$
\left\langle H_{\mathrm{n}}\right\rangle=\frac{2 \pi \chi \zeta}{\sigma a^{2}}
$$

Previous studies have shown that the constant mean curvature model is the most appropriate model for describing the curved interfaces of the inverse bicontinuous phases, ${ }^{34}$ therefore $a$ can be described by eqn (3) and the surface averaged mean curvature, defined by the CMCM, is:

$$
\left\langle H_{\mathrm{n}}\right\rangle_{\mathrm{CMCM}}=\frac{A_{\mathrm{n}} \sum_{i=0} \xi_{i}\left[\left(\frac{V_{\mathrm{n}}}{V}\right)\left(1-\phi_{\mathrm{w}}\right)\right]^{2 i+1}}{2 V \sum_{i=0} \sigma_{i}\left[\frac{V_{\mathrm{n}}}{V}\left(1-\phi_{\mathrm{w}}\right)\right]^{2 i}}
$$

By determining the effects of pressure and temperature on the lattice parameter and consequently the pivotal surface, it is possible to make semi-quantitative statements about the effect of pressure, temperature or water content on the stability of the $\mathrm{Q}_{\text {II }}^{\mathrm{G}}$ phase.

\section{Data fitting}

Fitting temperature dependent data. Swelling data obtained from SAXs experiments at atmospheric pressure were fitted to the PIM and the CMCM respectively using Mathematica ${ }^{\circledR}$ 9. Data points with a standard deviation greater than 1.5 were removed, to reduce the error from the fits which may arise from erroneous data points, and the model was then refitted to obtain the pivotal surface parameters $\left\langle A_{\mathrm{n}}\right\rangle$ and $\left\langle\frac{V_{\mathrm{n}}}{V}\right\rangle$ (Fig. 2). To reduce the experimental error incurred by preparation of large volumes of limited hydrated monolinolein, the models were fitted to a recalculated water content, $\varnothing_{\mathrm{w}}^{\prime}$, to obtain $\left\langle A_{\mathrm{n}}\right\rangle$ and $\left\langle\frac{V_{\mathrm{n}}}{V}\right\rangle$. The recalculated water content for each sample was obtained from the corresponding lattice parameter at 1 bar and $30{ }^{\circ} \mathrm{C}$ using eqn (1) and (2) where $A_{\mathrm{n}}, V_{\mathrm{n}}$ and $V$ were obtained by fitting the models to isobaric data obtained at $30{ }^{\circ} \mathrm{C}$ as the water content at different pressures and temperatures is constant for a homogeneous sample. Whilst eliminating the error between the actual water content and the expected water content this statistical treatment meant that the swelling data at constant temperature was constrained to a fixed geometry, therefore it is only possible to determine the qualitative effect of pressure on the pivotal surface.

\section{Results and discussion}

Swelling data obtained from SAXS experiments at constant pressure, from $10^{\circ} \mathrm{C}$ to $35^{\circ} \mathrm{C}$ and recalculated water contents at constant temperature, $30^{\circ} \mathrm{C}$ from 1 bar to 600 bar were fit to the PIM and CMCM using the molecular volume, $V$, obtained from density measurements (ESI Table S1 $\dagger$ ) to obtain the pivotal surface parameters $A_{\mathrm{n}}$ and $V_{\mathrm{n}}$ as a function of temperature and pressure.

The results showed that there was a good fit to isobaric data with a $R^{2} \geq 0.999999$ and to isothermal data where $R^{2} \geq 0.999$ confirming that the models are a valid description of the pivotal surface in the $\mathrm{Q}_{\mathrm{II}}^{\mathrm{G}}$ phase under these conditions. The precise interfacial geometry of the bicontinuous cubic phases is not known, but it has been modelled in two ways ${ }^{26}$ - an interface of uniform thickness (the parallel interface model) and one with constant mean curvature (the constant mean curvature model). The parallel interface model makes geometric calculations straightforward, but has the unfortunate characteristic that bicontinuous cubic phases, such as $\mathrm{Q}_{\mathrm{II}}^{\mathrm{G}}$, $\mathrm{Q}_{\mathrm{II}}^{\mathrm{D}}$ and $\mathrm{Q}_{\mathrm{II}}^{\mathrm{P}}$, which are related by a Bonnet transformation, exhibit energetic degeneracy for any energetic term that depends on interfacial geometry. ${ }^{35}$ This energetic degeneracy does not exist when the interface has constant mean curvature and it has been shown that the qualitative characteristics of the phase behaviour of the three bicontinuous cubic phases are replicated. For example, replication of the phase sequence from $\mathrm{Q}_{\mathrm{II}}^{\mathrm{G}} \rightarrow \mathrm{Q}_{\mathrm{II}}^{\mathrm{D}} \rightarrow \mathrm{Q}_{\mathrm{II}}^{\mathrm{P}}$ with increasing water content and negative phase boundaries are observed. ${ }^{34}$ Despite this, this geometric model has its own limitations - the interfaces become self-intersecting at reduced hydrations, where the mesophases are still found to exist in nature. ${ }^{36,37}$ Within these limitations both models are able to give us insight into the behaviour of these lyotropic structures as thermodynamic conditions are varied. Our studies showed that comparisons between the geometric fits showed no difference, within error, in the pivotal surface parameters between $10{ }^{\circ} \mathrm{C}$ and $35{ }^{\circ} \mathrm{C}$ 

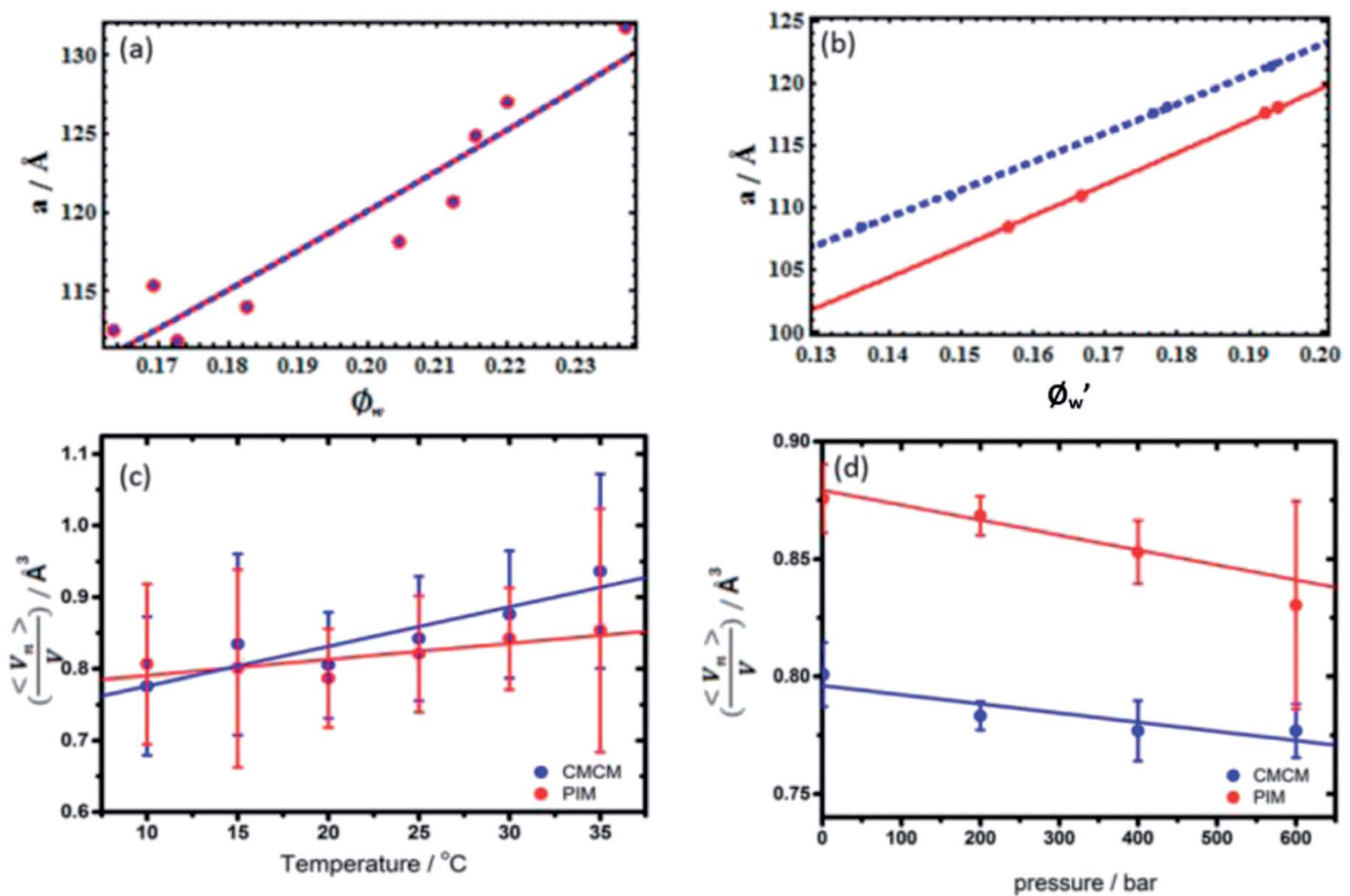

Fig. 2 Determining the location of the pivotal surface as a function of pressure and temperature. (a) Example of CMCM (blue) and PIM (red) fits to isobaric experimental swelling data at $25^{\circ} \mathrm{C}$; (b) example of CMCM (blue) and PIM (red) fits to recalculated water contents at $30{ }^{\circ} \mathrm{C}$ at 400 bar. Weighted linear fits of isobaric and isothermal $\frac{V_{\mathrm{n}}}{V}$ show (c) a shift towards headgroup region with increasing temperature and (d) a shift towards the bilayer midplane with increasing pressure from the CMCM (blue) and PIM (red).

(Fig. 3 and S1†) and this is attributed to small contributions of chain stretching to the energy of the $\mathrm{Q}_{\text {II }}^{\mathrm{G}}$ phase in monolinolein when $\emptyset_{\mathrm{w}}$ lies between 0.12 and 0.25 . These results suggest that under these conditions, both geometric models are suitable for determining the pivotal surface parameters for the $\mathrm{Q}_{\mathrm{II}}^{\mathrm{G}}$ phase, indicating that there are contributions from curvature elastic stress and packing frustration to the energetics of the $\mathrm{Q}_{\mathrm{II}}^{\mathrm{G}}$ phase in limited hydration monolinolein. Determining the precise contributions of the curvature elastic energy and packing frustration is a non-trivial matter which could be achieved if the error in experiment was less than the difference in contributions from curvature stress and packing frustration. At atmospheric pressure and $25{ }^{\circ} \mathrm{C},\left(\frac{V_{\mathrm{n}}}{V}\right)_{\mathrm{CMCM}}=0.83 \pm 0.1$ which positions the pivotal surface between carbon 1 and 2 of the acyl chain. This is comparable to $\left(\frac{V_{\mathrm{n}}}{V}\right)$ obtained using the PIM in monoolein ${ }^{23}$ and didodecyl-b-D-glucopyranosyl ${ }^{38}$ of 0.76 and 0.83 respectively and corresponds to the position in the acyl chain where the lateral compressibility is at its lowest and therefore where the interface will pivot. Our results show that the position of the pivotal surface is linearly dependent on temperature and pressure. Increasing the temperature shifts the pivotal surface away from the bilayer midplane by $0.2 \%$ $\mathrm{K}^{-1}$ and increasing the pressure moves the pivotal surface towards the bilayer midplane by approximately $6 \% \mathrm{kbar}^{-1}$.
Previous studies showed that $\left(\frac{V_{\mathrm{n}}}{V}\right)$ was constant with temperature ${ }^{23}$ and deconvolution of the effects of pressure and temperature on the pivotal surface were attributed to greater precision in the experimental data afforded by technological advances in bench top X-rays and $3^{\text {rd }}$ generation synchrotron sources. The sensitivity of the location of the pivotal surface to changes in temperature and pressure can be understood by considering the lateral stresses within the monolayer as the thermodynamic parameters are altered. For example, increasing the temperature increases the positive lateral stresses in the acyl chain region due to an increase in the number of trans-gauche rotamers in the hydrocarbon chain region whilst increasing the pressure leads to a decrease in positive lateral stresses as the hydrocarbon chain is compressed. Indeed, calculation of the compressibility of the acyl chain region between the pivotal surface and the bilayer midplane and the pivotal surface $\left(B_{\mathrm{ad}}\right)$ from experimental data (see ESI†) gave $7.15 \times 10^{-10} \mathrm{~Pa}^{-1}$ (CMCM) which is comparable to the adiabatic compressibility of linoleic acid, $6.90 \times 10^{-10} \mathrm{~Pa}^{-1}$ at $65^{\circ} \mathrm{C}$, obtained by ultrasound experiments. ${ }^{15}$ This indicates that the compressibility in the acyl chain, affected by changes in temperature and pressure, will lead to changes in the lateral stresses within the bilayer. Consequently the pivotal surface will move transverse to the bilayer midplane to maintain a balance of forces on either side of the pivotal surface. 

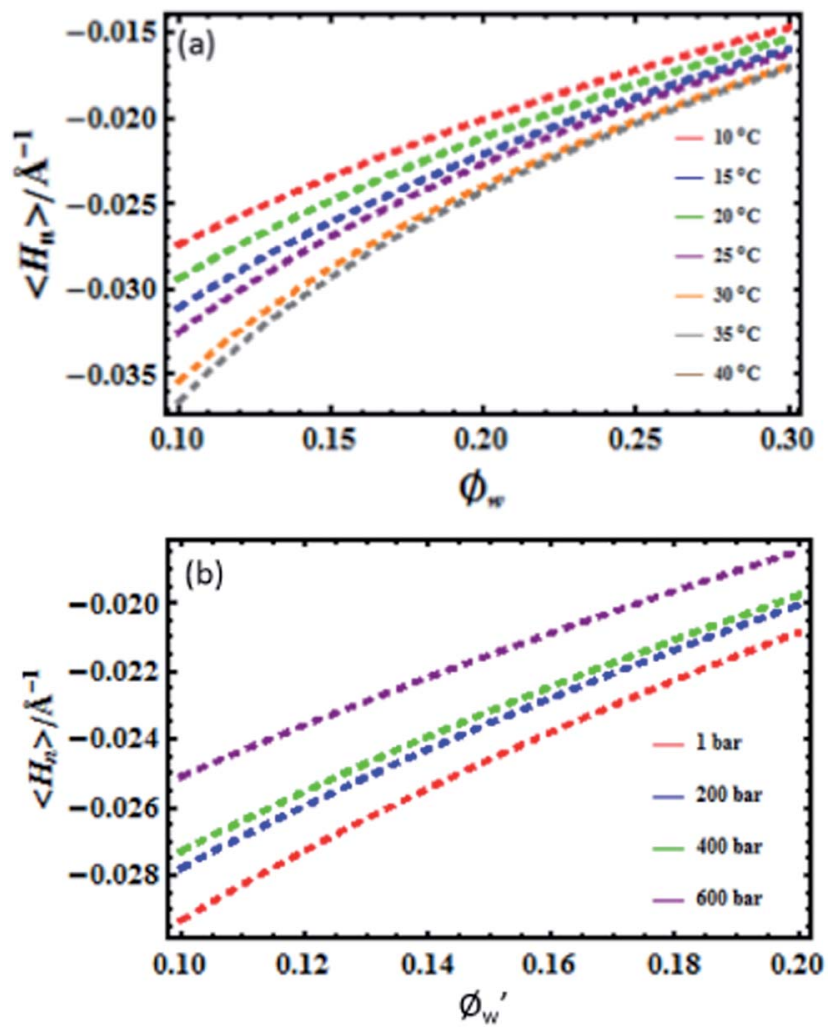

Fig. 3 Surface averaged mean curvature calculated for bilayers constrained to the constant mean curvature interface using the experimentally determined pivotal surface parameters (a) $H_{n}$ becomes more negative with increasing temperature, dashed, red $-10{ }^{\circ} \mathrm{C}$, blue $-15^{\circ} \mathrm{C}$, green-20 ${ }^{\circ} \mathrm{C}$, purple- $25^{\circ} \mathrm{C}$, orange $-30{ }^{\circ} \mathrm{C}$, grey- $35^{\circ} \mathrm{C}$, brown- $40{ }^{\circ} \mathrm{C}$ (b) $H_{n}$ becomes less negative with increasing pressure, red-1 bar, blue200 bar, green-400 bar, purple-600 bar.

In order to determine the effects of pressure and temperature on the curvature elastic energy, the pivotal surface parameters obtained from experimental data were used to calculate $H_{\mathrm{n}}$ as a function of water content, pressure and temperature, using the constant mean curvature model. The results showed a non-linear dependence on $H_{\mathrm{n}}$ with a decreasing negative curvature with increasing water content at all measured temperatures and pressures. Assuming that $\left\langle K_{\mathrm{n}}\right\rangle$ is minimized, a decrease in $H_{\mathrm{n}}$ with increasing negative curvature describes a reduction in the asymmetric molecular deformation of the lipid shape. This is consistent with previous results which underpins characteristic phase transitions from $\mathrm{Q}_{\mathrm{II}}^{\mathrm{G}}$ to $\mathrm{Q}_{\mathrm{II}}^{\mathrm{D}}$ to $\mathrm{Q}_{\mathrm{II}}^{\mathrm{P}}$ with increasing water content for this class of lipids, ${ }^{34}$ and shows that the quadratic dependence of curvature elastic energy on $H_{\mathrm{n}}$ removes the energetic degeneracy between the three inverse bicontinuous cubic phases. Increasing the temperature caused $H_{\mathrm{n}}$ to increase in magnitude, whilst increasing the pressure led to a decrease in magnitude in $H_{\mathrm{n}}$. These qualitative trends are consistent with observations from experimental phase diagrams for monolinolein where the more curved phase, $\mathrm{Q}_{\mathrm{II}}^{\mathrm{G}}$, was destabilized at higher pressure in favour of the less curved lamellar phase whilst increasing the temperature stabilized the more curved phases over the lamellar phase. ${ }^{7}$
$H_{\mathrm{n}}$ shows an anomalous trend with increasing temperature, where $H_{\mathrm{n}}^{20}{ }^{\circ} \mathrm{C}>H_{\mathrm{n}}^{15}{ }^{\circ} \mathrm{C}$ (Fig. 3a); this is attributed to the greater error in $H_{\mathrm{n}}^{15}{ }^{\circ} \mathrm{C}$ compared to $H_{\mathrm{n}}^{20}{ }^{\circ} \mathrm{C}$ (Fig. $\mathrm{S} 4 \dagger$ ) and is due to the accuracy of $A_{\mathrm{n}}$ and $V_{\mathrm{n}}$ obtained by model fitting. The curvature elastic energy of a mesophase is dependent on the accuracy of the pivotal surface parameters and thus the measured lattice parameters which are a reflection of changes to the molecular shape, therefore the curvature elastic energy is a subtle function of the molecular shape. In addition, experimental errors, from loss of water or lipid during sample preparation will lead to errors in the water volume fraction, consequently affecting the accuracy of $A_{\mathrm{n}}$ and $V_{\mathrm{n}}$.

\section{Conclusions}

By fitting isothermal and isobaric swelling data with models which constrain the interfacial geometry of a lipid bilayer, we have shown for the first time the effect of pressure and temperature on the location of the pivotal surface and shown that the model fits the data consistently and provides a selfconsistent explanation for the swelling behavior in the $\mathrm{QII}_{\mathrm{G}}^{\mathrm{G}}$ phase. The lattice parameter of the unit cell is directly related to the molecular shape at a given pressure and temperature, consequently the pivotal surface parameters are a result of the lateral stress profiles at any pressure and temperature. Therefore pivotal surface parameters at certain pressures and temperatures give predictive power of the swelling behavior in the $\mathrm{QII}_{\mathrm{II}}^{\mathrm{G}}$ phase with reasonable accuracy. Qualitative trends in the mean curvature as a function of water content, temperature and pressure are consistent with experimental phase behavior, affirming that within the precision of our measurements the geometric models replicate swelling behavior well. These observations reinforce the importance of these geometric models for describing curved bilayers and are a good basis for a full theoretical description of lyotropic phase behavior.

Our experiments so far have been limited to the $\mathrm{Q}_{\mathrm{II}}^{\mathrm{G}}$ phase, and further investigations on the pivotal surface parameters in the $Q_{I I}^{D}$ and $Q_{I I}^{P}$ phase are required for a full discussion on the accuracy of the geometric models for obtaining the pivotal surface parameters in the inverse bicontinuous cubic phases. Furthermore, in order to disentangle the contributing forces to the energetics of the inverse bicontinuous cubic phases we require a deeper understanding of the effect of changing the lateral stress profiles on the curvature elasticity.

\section{Acknowledgements}

This work was supported by EPSRC Platform Grant EP/G00465X and by an EPSRC DTA studentship awarded to T-YDT. We acknowledge Diamond Light Source (UK) and the European Synchrotron Radiation Facility (Grenoble, France) for the provision of synchrotron radiation facilities and we would like to thank Dr Claire Pizzey (beamline I22, DLS), and Dr Michael Sztucki (beamline ID02, ESRF) for their assistance during the synchrotron experiments. 


\section{References}

1 A. H. Schoen, Report D-5541, NASA, Washington DC, 1970.

2 H. Schwarz, Gesammelte Mathematische Abhandlungen, Springer, Berlin, 1890.

3 D. F. Evans and H. Wennerstrom, The colloidal domain, where physics, chemistry, biology and technology meet, 1999.

4 G. L. Kirk, S. M. Gruner and D. L. Stein, Biochemistry, 1984, 23, 1093-1102.

5 E. S. Lutton, J. Am. Oil Chem. Soc., 1965, 42, 1068-1070.

6 M. Caffrey, J. Briggs and H. Chung, J. Phys. II, 1996, 6, 723751.

7 T. Y. D. Tang, N. J. Brooks, C. Jeworrek, O. Ces, N. J. Terrill, R. Winter, R. H. Templer and J. M. Seddon, Langmuir, 2012, 28, 13018-13024.

8 R. Winter, Biophys. J., 2002, 82, 153A.

9 R. Winter, Curr. Opin. Colloid Interface Sci., 2001, 6, 303-312.

10 P. M. Duesing, J. M. Seddon, R. H. Templer and D. A. Mannock, Langmuir, 1997, 13, 2655-2664.

11 C. V. Kulkarni, T.-Y. Tang, A. M. Seddon, J. M. Seddon, O. Ces and R. H. Templer, Soft Matter, 2010, 6, 3191-3194.

12 G. Cevc, A. Watts and D. Marsh, Biochemistry, 1981, 20, 49554965.

13 B. J. Boyd, D. V. Whittaker, S.-M. Khoo and G. Davey, Int. J. Pharm., 2006, 309, 218-226.

14 J. Bender, M. B. Ericson, N. Merclin, V. Iani, A. Rosen, S. Engstrom and J. Moan, J. Controlled Release, 2005, 106, 350-360.

15 G. O. Hustad, T. Richardson, W. C. Winder and M. P. Dean, Chem. Phys. Lipids, 1971, 7, 61-74.

16 N. J. Brooks, B. L. L. E. Gauthe, N. J. Terrill, S. E. Rogers, R. H. Templer, O. Ces and J. M. Seddon, Rev. Sci. Instrum., 2010, 81, 064103.

17 R. G. Kimber, A. B. Walker, G. E. Schröder-Turk and D. J. Cleaver, Phys. Chem. Chem. Phys., 2010, 12, 844-851.

18 V. Cherezov, J. Mol. Biol., 2006, 357, 1605-1618.
19 P. Nollert, H. Qiu, M. Caffrey, J. P. Rosenbusch and E. Landau, FEBS Lett., 2001, 179-186.

20 G. Rummel, A. Hardmeyer, C. Widmer, M. Chiu, P. Nollert, K. Locher, I. Pedruzzi, E. Landau and J. Rosenbusch, J. Struct. Biol., 1998, 182-191.

21 C. Leal, N. F. Bouxsein, K. K. Ewert and C. R. Safinya, J. Am. Chem. Soc., 2010, 132, 16841-16847.

22 R. H. Templer, J. M. Seddon and N. A. Warrender, Biophys. Chem., 1994, 49, 1-12.

23 R. H. Templer, Langmuir, 1995, 11, 334-340.

24 S. M. Gruner, J. Phys. Chem., 1989, 93, 7562-7570.

25 S. T. Hyde, J. Phys. Chem., 1989, 93, 1458-1464.

26 D. M. Anderson, S. M. Gruner and S. Leibler, Proc. Natl. Acad. Sci. U. S. A., 1988, 85, 5364-5368.

27 K. Grosse-Brauckmann, J. Colloid Interface Sci., 1997, 187, 418-428.

28 K. Grosse-Brauckmann, Interface Focus, 2012, 2, 582-588.

29 P. Padmanabhan, F. J. Martinez-Veracoechea, J. C. Araque and F. A. Escobedo, J. Chem. Phys., 2012, 136.

30 C. Czeslik, R. Winter, G. Rapp and K. Bartels, Biophys. J., 1995, 68, 1423-1429.

31 O. Reis and R. Winter, Langmuir, 1998, 14, 2903-2909.

32 J. M. Seddon, A. M. Squires, C. E. Conn, O. Ces, A. J. Heron, X. Mulet, G. C. Shearman and R. H. Templer, Philos. Trans. $R$. Soc., A, 2006, 364, 2635-2655.

33 R. H. Templer, J. M. Seddon, P. M. Duesing, R. Winter and J. Erbes, J. Phys. Chem. B, 1998, 102, 7262-7271.

34 G. C. Shearman, O. Ces and R. H. Templer, Soft Matter, 2010, 6, 256-262.

35 U. S. Schwarz and G. Gompper, Langmuir, 2001, 17, 20842096.

36 Y. Deng, Z. A. Almsherqi, M. M. L. Ng and S. D. Kohlwein, Trends Cell Biol., 2010, 20, 371-379.

37 T. Landh, FEBS Lett., 1995, 369, 13-17.

38 D. C. Turner, Z.-G. Wang, S. M. Gruner, D. A. Mannock and R. N. McElhaney, J. Phys. II, 1992, 2, 2039-2063. 